\title{
Template Matching Using Grey Wolf Optimizer with Lateral Inhibition
}

\author{
Sen Zhang ${ }^{1}$ Yongquan Zhou ${ }^{1,2}$ \\ ${ }^{1}$ College of Information Science and Engineering,Guangxi University for Nationalities, Nanning 530006, China \\ ${ }^{2}$ Guangxi High School Key Laboratory of Complex System and Computational Intelligence, Nanning 530006, China \\ e-mail: yongquanzhou@126.com
}

\begin{abstract}
In this paper, a hybrid method of grey wolf optimizer (GWO) and lateral inhibition (LI) is proposed to solve complicated template matching problems. The proposed template matching technique is called LI-GWO. GWO is a new meta-heuristic algorithm inspired by the hunting behavior and social leadership of grey wolves in nature. In addition, lateral inhibition mechanism has been verified to have good effects on image edge extraction and image enhancement. So we employ lateral inhibition for image pre-processing. LI-GWO combines both advantages of GWO and literal inhibition and makes better performance. Series of comparative experimental results show that the proposed method achieves the best balance in comparison to other algorithms based on lateral inhibition in terms of estimation accuracy and the computational cost.
\end{abstract}

Keywords: grey wolf optimizer (GWO); lateral inhibition (LI); image process; template matching.

\section{Introduction}

Template matching refers to recognizing predefined template images in a source image. It plays an important role in face recognition [1], pulmonary nodules detection [2], handwriting identification [3], and road detection [4] over the past few decades.

The classical template matching method, which compares the template with the captured image pixel by pixel, is based on gray cross-correlation measurement. Nowadays, many template matching algorithms have been developed [5-8], and they can be classified as the intensity-based method or the feature-based method $[9,10]$. The intensity-based method can be regarded as an optimization process of finding the maximum similar degree between the template and the original image. On the other hand, feature-based method matches on the basis of image feature such as border, unique points, texture, entropy, and energy. The performance of feature-based method greatly depends on the quality and stability of selected dynamic features, and in addition, it varies in different situations. Compared with the feature-based method, the intensity-based method provides better performance and widely used as it is independent from extensive feature extractions and has superior ability to restrain noise. However, the cost of computing time in template matching is tremendous. Thus, solving the problems requires effective optimization techniques.

Recently, many global optimization algorithms such as particle swarm optimization (PSO) [10, 11], bat algorithm (BA) [12], artificial bee colony (ABC) [13-15], biogeography-based optimization (BBO) [16], states of matter search (SMS) algorithm [17], and imperialist competitive algorithm (ICA) [18], have been proposed for this template matching problem. Although these algorithms aim to reduce the time cost of global searching, they cannot avoid the derivation of suboptimal matching results. Therefore, it needs a new way to modify these existing evolutionary algorithms so as to achieve a better balance considering the estimation accuracy and the computational cost. In this paper, grey wolf 
optimizer (GWO) is presented to solve the template matching problem.

Grey wolf optimizer (GWO) [19] is a new optimization algorithm which simulates hunting behavior and social leadership of grey wolves in nature. Numerical comparisons showed that the superior performance of GWO is competitive to that of other population-based algorithms. Because it is simple, easy to implement, and has fewer control parameters, GWO has caused much attention and has been used to solve a number of practical optimization problems [20-28].

In addition, lateral inhibition (LI) approach is utilized to preprocess the original and template images before using GWO for matching. The hybrid method is named grey wolf optimizer based on lateral inhibition (LI-GWO). Lateral inhibition is firstly discovered by Hartline et al. in studying of the eyes of limulus [29]. By numerous researches, lateral inhibition mechanism has been certified to be effective in image enhancement and edge extraction and it can increase the accuracy of image matching [30]. The proposed method LI-GWO combines the advantages of both GWO and lateral inhibition, proved better performance comparing to LI-BBO, LI-BA, LI-SMS, and LI-ICA.

The rest of the paper is structured as follows. Section 2 discusses the principles of GWO. Section 3 describes lateral inhibition mechanism. Section 4 details the implementation procedures of the hybrid method LI-GWO. Then, several cases of comparative experiments and further discussions have been conducted in Section 5. Finally, Section 6 concludes the study and advises some directions for future studies.

\section{The basic principle of grey wolf optimizer}

Grey wolf optimizer [19] is a new meta-heuristic optimization method, which is proposed by Mirjalili et al. and based on the simulation of the hunting behavior and social leadership of grey wolves in nature. It is similar to other meta-heuristics that it initials a population of wolves randomly based on the upper and lower bounds of the variables. During every iteration, the first three best wolves are considered as alpha $(\alpha)$, beta $(\beta)$, and delta $(\delta)$, who lead other wolves toward promising zones of the search space. The rest of grey wolves are thought of as omega $(\omega)$ who update their positions encircling $\alpha, \beta$, or $\delta$ as follows :

$$
\begin{aligned}
& \vec{D}=\left|\vec{C} \cdot \vec{X}_{p}(t)-\vec{X}(t)\right| \\
& \vec{X}(t+1)=\vec{X}_{p}(t)-\vec{A} \cdot \vec{D}
\end{aligned}
$$

Where $t$ is the current iteration, $\vec{C}=2 \cdot \vec{r}_{2}, \vec{A}=2 \vec{a} \cdot \vec{r}_{1}-\vec{a}, \vec{X}_{p}$ is the position vector of the prey, $\vec{X}$ is the position vector of a grey wolf, $\vec{a}$ is gradually decreased from 2 to 0 , and $r_{1}, r_{2}$ are random numbers in $[0,1]$.

It should be noted here that during optimization the $\omega$ wolves update their positions encircling $\alpha, \beta$, and $\delta$. So each $\omega$ wolf can reposition relative to $\alpha, \beta$, and $\delta$ concurrently as follows :

$$
\begin{gathered}
\vec{D}_{\alpha}=\left|\vec{C}_{1} \cdot \vec{X}_{\alpha}-\vec{X}\right|, \vec{D}_{\beta}=\left|\vec{C}_{2} \cdot \vec{X}_{\beta}-\vec{X}\right|, \vec{D}_{\delta}=\left|\vec{C}_{3} \cdot \vec{X}_{\delta}-\vec{X}\right| \\
\vec{X}_{1}=\vec{X}_{\alpha}-\vec{A}_{1} \cdot\left(\vec{D}_{\alpha}\right), \vec{X}_{2}=\vec{X}_{\beta}-\vec{A}_{2} \cdot\left(\vec{D}_{\beta}\right), \vec{X}_{3}=\vec{X}_{\delta}-\vec{A}_{3} \cdot\left(\vec{D}_{\delta}\right) \\
\vec{X}(t+1)=\frac{\vec{X}_{1}+\vec{X}_{2}+\vec{X}_{3}}{3}
\end{gathered}
$$


Where $\vec{X}_{\alpha}, \vec{X}_{\beta}$, and $\vec{X}_{\delta}$ indicate the positions of the alpha, beta, and delta, respectively. And $\vec{C}_{1}, \vec{C}_{2}, \vec{C}_{3}$ and $\vec{A}_{1}, \vec{A}_{2}, \vec{A}_{3}$ are all random vectors, $\vec{X}$ is the position of the current solution, and $t$ is the number of iterations.

According to [19], the parameters A and c oblige the GWO to explore and exploit the search space. With decreasing $A$, half of the iterations are devoted to exploration (when $|A|>1$ ) and the rest are dedicated to exploitation (when $|A|<1$ ). We can know from the above that the $\vec{C}$ vector contains random values in $[0,2]$. This component provides random weights for prey in order to stochastically emphasize $(C>1)$ or deemphasize $(C<1)$ the effect of prey in defining the distance in Eq.(1). This assists GWO to show a more random behavior during the course of optimization and favors exploration and local optima avoidance. More detailed information about the GWO can be found in [19]. Based on these approximations and idealization, the basic steps of grey wolf optimizer can be made a summing up in Algorithm 1.

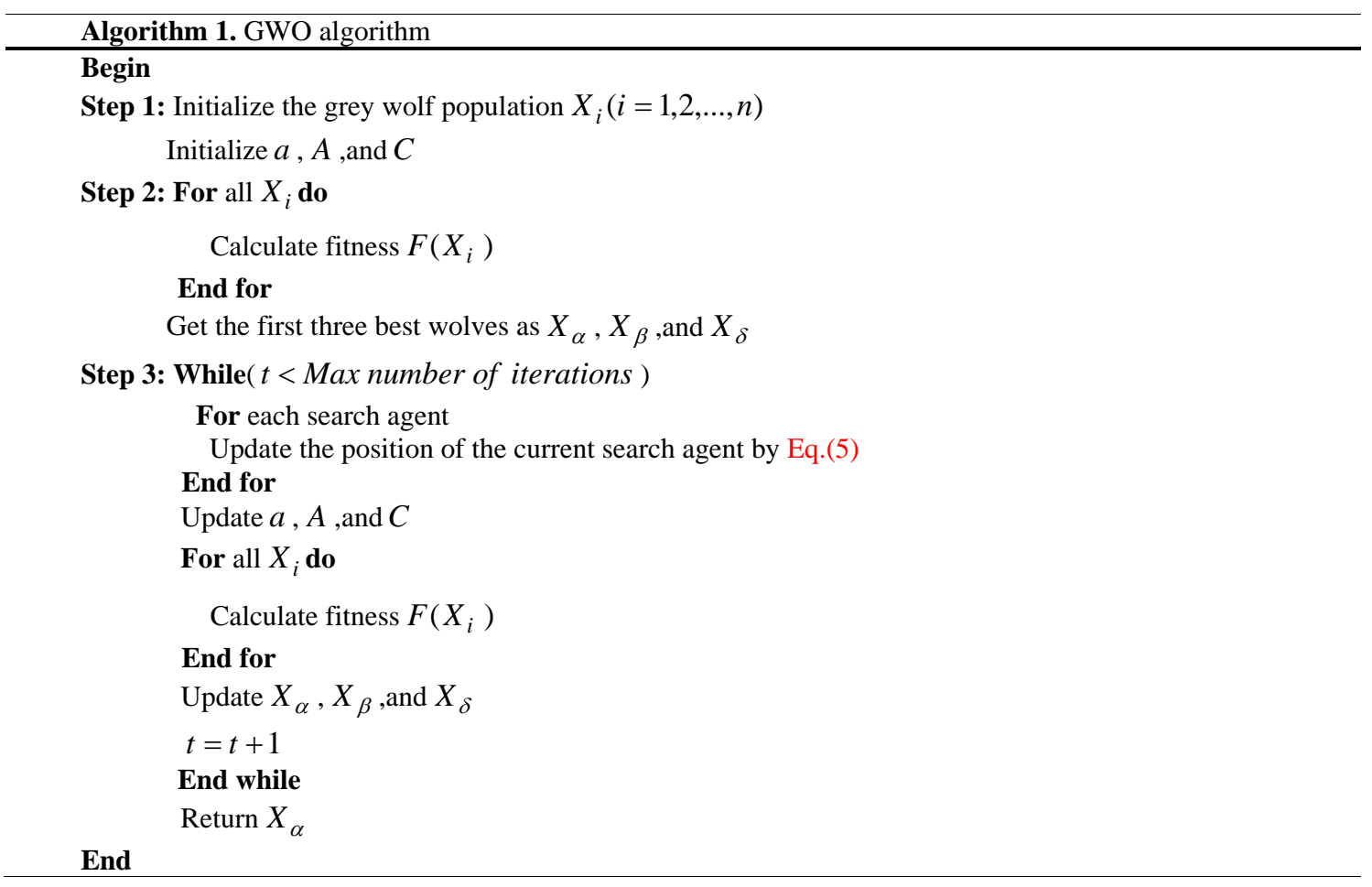

\section{Lateral inhibition mechanism}

The lateral inhibition mechanism was first discovered and confirmed by Hartline et al. when they conducted an electrophysiology experiment on the limulus' vision [29]. They found that every microphthalmia of limulus' ommateum is regarded as a receptor that is restricted by its adjacent receptors, and the inhibited effect is mutual and spatially summed [10]. The nearer the close receptors are from each other, the stronger the inhibited effect is.

In retinal images, intensively excited receptors in illuminatingly light area inhibit those in dark area more strongly than the latter to the former [30]. Therefore, lateral inhibition effectively increases the contrast and the distortion of sensory information. By this means, the important information of the 
image namely the edge of image including the intensity gradient in retinal image and the important characters of vision scene is strongly strengthened. In this paper, we apply the mechanism to preprocess the template and the original images in order to improve the spatial resolution and increase the efficiency and accuracy of the template matching.

The classical lateral inhibition model is shown as follows:

$$
r_{p}=e_{p}+\sum_{j=1}^{n} k_{p, j}\left(r_{j}-r_{p, j}\right), p=1,2, \ldots, n ; j \neq p
$$

In order to apply this mechanism to image processing, the model is modified into a two-dimensional and gray form. Gray value of the pixel $(m, n)$ in image is expressed with the following equation:

$$
R(m, n)=I_{0}(m, n)+\sum_{i=-M}^{M} \sum_{j=-N}^{N} \alpha_{i, j} I_{0}(m+i, n+j)
$$

where $\alpha_{i, j}$ is the lateral inhibition coefficient of the pixel $(i, j)$ to the central pixel, $I_{0}(m, n)$ represents the original gray value of pixel $(m, n), R(m, n)$ is the gray value of pixel $(m, n)$ after being processed by lateral inhibition, and $M \times N$ denotes the scale of the receptive field.

The size of receptive field chosen in this paper is $5 \times 5$. Then the competing coefficient of the lateral inhibition network is:

$$
\begin{aligned}
R(m, n)= & \alpha_{0} \times I_{0}(m, n)+\alpha_{1}\left[\sum_{i=-1}^{1} \sum_{j=-1}^{1} I_{0}(m+i, n+j)-I_{0}(m, n)\right] \\
& +\alpha_{2}\left[\sum_{i=-2}^{2} \sum_{j=-2}^{2} I_{0}(m+i, n+j)-\sum_{i=-1}^{1} \sum_{j=-1}^{1} I_{0}(m+i, n+j)\right]
\end{aligned}
$$

Because the vision nerve cells are situated at the same input plane and the competing coefficients are close to zero, the template of lateral inhibition coefficient satisfies:

$$
\alpha_{0}+8 \alpha_{1}+16 \alpha_{2}=0
$$

In this paper, we choose $\alpha_{0}=1, \alpha_{1}=-0.075, \alpha_{2}=-0.025$ to form the following matrix as the modulus.

$$
U=\left[\begin{array}{ccccc}
0.025 & 0.025 & 0.025 & 0.025 & 0.025 \\
0.025 & 0.075 & 0.075 & 0.075 & 0.025 \\
0.025 & 0.075 & 1 & 0.075 & 0.025 \\
0.025 & 0.075 & 0.075 & 0.075 & 0.025 \\
0.025 & 0.025 & 0.025 & 0.025 & 0.025
\end{array}\right]
$$

The modulus template $U$ is combined with $R(m, n)$ and then a new gray scale of the image is obtained. Finally, the image's edge is extracted by the following equation.

$$
F(m, n)= \begin{cases}0 & R(m, n) \leq T \\ 255 & R(m, n)>T\end{cases}
$$

where $T$ is a user-defined threshold value according to practical situations, $F(m, n)$ is the final obtained gray value of pixel $(m, n)$.

\section{Hybrid GWO and lateral inhibition}




\subsection{The fitness function of LI-GWO}

In this paper, the proposed LI-GWO is introduced to conduct the searching task. The fitness function is defined to calculate the fitness of every grey wolf according to different situations and real practices. Template matching based on gray cross-correlation measurement has strong ability to suppress noise and the performance of simple calculation, while it is time-consuming [11]. Our work follows the custom of criterion selection as in references $[11,16]$, the fitness value of template matching is shown in Fig. 1.

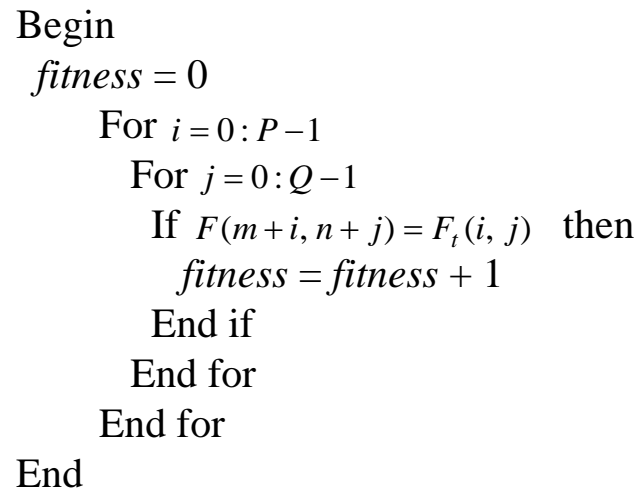

Fig. 1 The method of calculating the fitness in LI-GWO

where fitness is the similar degree between the template and the pattern whose starting point is pixel $(m, n)$ in the original image. So the maximum fitness denotes the best solution of the template matching problem. The similarity measurement can reduce the calculation time and is easy to program. $(m+i, n+j)$ and $(i, j)$ respectively represent the coordinates of the pixel in the original image and the template image. $F(m+i, n+j)$ and $F_{t}(i, j)$ are the gray values of pixel $(m+i, n+j)$ and pixel $(i, j)$ processed by Eq. (11). If $M \times N$ is the size of the original image and $P \times Q$ is the size of the template image, the scope of the coordinates in the original image for matching is $1 \leq m \leq M-P+1$ and $1 \leq n \leq N-Q+1$. Therefore, we define low $_{1}=1, \quad$ low $_{2}=1, u p_{1}=M-P+1$, $u p_{2}=N-Q+1$ in our experiments.

\subsection{The procedure of LI-GWO}

The proposed LI-GWO combines the efficiency of GWO and the accuracy of lateral inhibition mechanism. It is proved to have good performance by the test in template matching. The process of the method is described as follows:

Step 1: Image pre-processing.

We obtain the original image and the template image, and we transform both of them into gray scale format. Then, we filter images to suppress noises and apply lateral inhibition mechanism to preprocess these images according to Eqs. (8)-(11) and save the new matrixes of images.

Step 2: Initialize the LI-GWO parameters.

We initialize the size of the population $N$, the dimension of the problem $D$, and the maximum function evolution number (MaxFEs). In this paper, $D$ is defined as two that is the dimension of images.

Step 3: Calculate the corresponding objective value for each wolf. 
Step 4: Choose the first three best wolves and save them as $\alpha, \beta$, and $\delta$.

Step 5: Update the position of the rest of the population ( $\omega$ wolves) using equations Eqs. (1)-(5).

Step 6: Update parameters $a, A$, and $C$.

Step 7: If the maximum iteration is not satisfied, go to step 3.

Step 8: Return the position of $\alpha$ as the best approximated optimum.

The detailed flow chart of LI-GWO approach for template matching is depicted in Fig. 2.
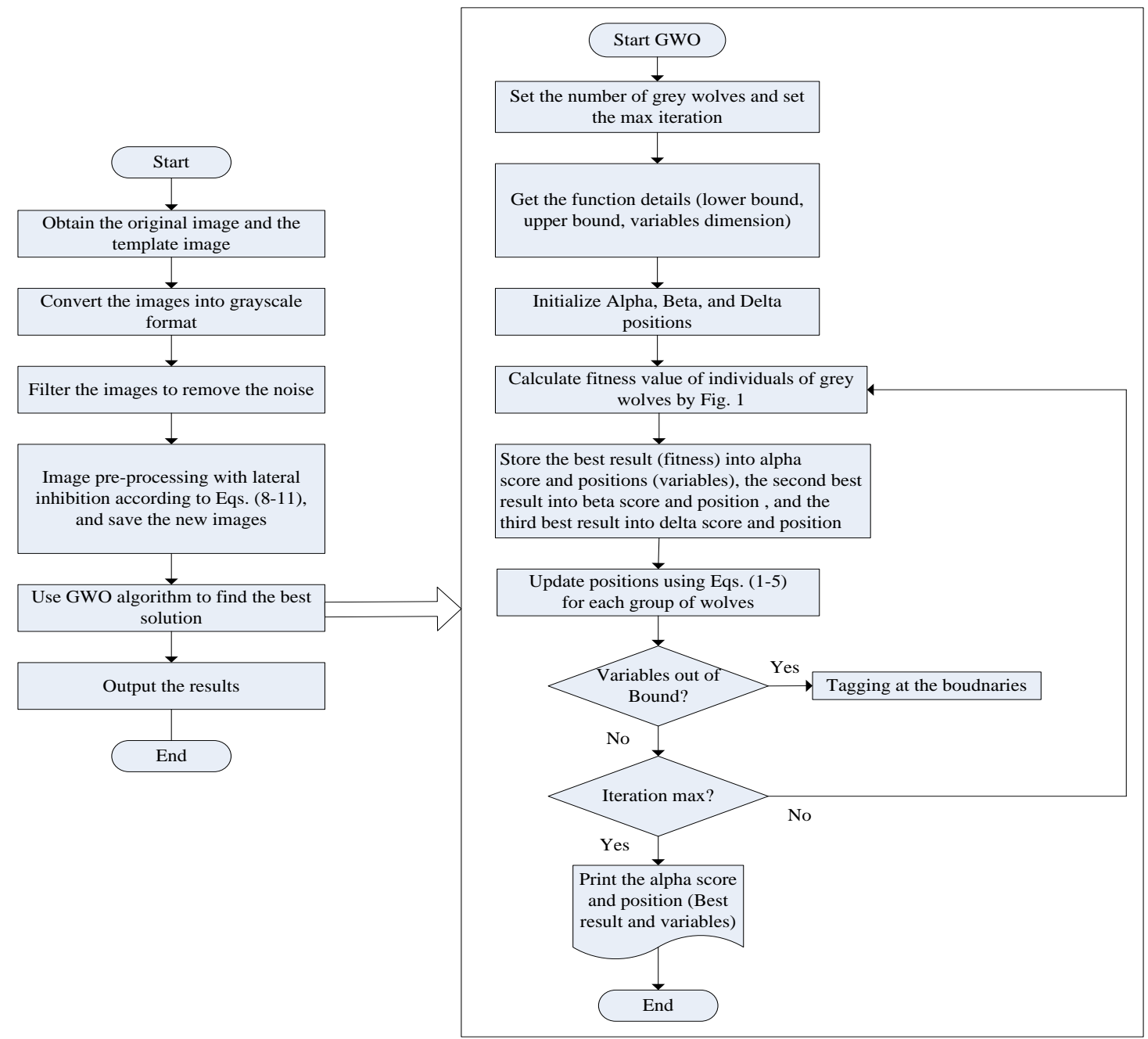

Fig. 2 Flow chart of LI-GWO for template matching

\section{Experimental results and discussions}

In this section, we verify the performance of our proposed algorithm through a series of comparative experiments with those produced by the BBO method [16], BA method [12, 31], SMS method [17], and ICA method [18]. Simulations have been executed across eight images that are shown in Figs. 3-10. All the simulations were carried out in a Matlab R2012a environment and executed on a 4-core Intel Core i5-4200U CPU with 8 GB RAM running at $4 \times 1.60 \mathrm{GHz}$ under Windows 8.1 operating system. In this test, the population size $N$ is set to 100. The maximum function evolution numbers (MaxFEs) is set to 300. Such the standard of stopping has been carefully selected to assure compatibility between similar works reported in the literature $[8,17]$. Initial parameters for each algorithm that is used in the comparison as follows: 


\section{(1) LI-BBO}

The parameters are set to: the maximum migration rates $I=1, E=1$, the maximum mutation probability $m_{\max }=0.001$, the number of elitisms keep $=3$.

(2) LI-BA

The parameters are set to: minimum frequency $f_{\min }=0$, maximum frequency $f_{\max }=2$, loudness of emission $A=0.25$, pulse rate $r=0.5$.

(3) LI-SMS

The parameters are set in gas $\rho \in[0.8,1], \beta=0.8, \alpha=0.8$ and $H=0.9$; in liquid $\rho \in[0.3,0.6]$ $\beta=0.4, \alpha=0.2$ and $H=0.2$; in solid $\rho \in[0,0.1], \beta=0.1, \alpha=0$ and $H=0$. Such values are the best parameter set for this algorithm according to [17].

(4) LI-ICA

The parameters are set to: NumOfCountries $=100$, NumOfImper $=10$, NumOfColony $=90$.

(5) LI-GWO:

The algorithm has been configured by using: $a=2-1 \times(2 /$ MaxCycle $)$ linearly decreased from 2 to 0 .

The threshold for image edge extracting was set to $T=105$. These experiments are designed to successfully match the template image to the original image. The coordinate of the template image fixed in the original image determines the success of matching. The experimental results are listed in Table 1. The results are averaged over 30 independent runs, and the best results are denoted in bold type. The average (Ave), average CPU Time, and correct rate are reported.

To improve the performance evaluation of evolutionary algorithms, statistical tests should be conducted [32]. In order to determine whether the results of LI-GWO differ from the best results of other algorithms in a statistical method, a nonparametric test which is known as Wilcoxon's rank-sum test $[33,34]$ is performed at $5 \%$ significance level. The calculated $p$ values in Wilcoxon's rank-sum test comparing LI-GWO and other algorithms over all the simulation cases are given in Table 2. Usually, $\quad p$ values $<0.05$ can be considered as sufficient evidence against the null hypothesis.

Table 1 Comparative optimization results obtained using LI-GWO and other algorithms

\begin{tabular}{|c|c|c|c|c|c|c|}
\hline image & Result & LI-BBO & LI-BA & LI-SMS & LI-ICA & LI-GWO \\
\hline \multirow[t]{3}{*}{1} & Ave & $4.6261 \mathrm{E}+03$ & $4.6777 \mathrm{E}+03$ & $4.7864 \mathrm{E}+03$ & $4.8410 \mathrm{E}+03$ & $4.8971 E+03$ \\
\hline & CPU Time(sec) & 5.4341 & 4.7026 & 4.9320 & 5.8163 & 4.2725 \\
\hline & Correct rate & $0.00 \%$ & $26.67 \%$ & $13.33 \%$ & $76.67 \%$ & $96.67 \%$ \\
\hline \multirow[t]{3}{*}{2} & Ave & $3.9660 \mathrm{E}+03$ & $3.7151 \mathrm{E}+03$ & $3.6230 \mathrm{E}+03$ & $3.8252 \mathrm{E}+03$ & $4.7440 \mathrm{E}+03$ \\
\hline & CPU Time(sec) & 6.8941 & 4.6264 & 5.7708 & 7.3351 & 5.0241 \\
\hline & Correct rate & $50.00 \%$ & $40.00 \%$ & $3.33 \%$ & $6.67 \%$ & $100.00 \%$ \\
\hline \multirow[t]{3}{*}{3} & Ave & $8.0566 \mathrm{E}+03$ & $7.1774 \mathrm{E}+03$ & $8.2047 \mathrm{E}+03$ & $7.9565 \mathrm{E}+03$ & $8.5070 E+03$ \\
\hline & CPU Time(sec) & 8.6817 & 7.9708 & 7.8190 & 10.6269 & 7.0072 \\
\hline & Correct rate & $43.33 \%$ & $26.67 \%$ & $40.00 \%$ & $56.67 \%$ & $100.00 \%$ \\
\hline \multirow[t]{3}{*}{4} & Ave & $2.0572 \mathrm{E}+03$ & $2.0843 \mathrm{E}+03$ & $2.2101 \mathrm{E}+03$ & $2.1178 \mathrm{E}+03$ & $2.9519 E+03$ \\
\hline & CPU Time(sec) & 5.5269 & 3.4608 & 4.1268 & 5.3741 & 3.0382 \\
\hline & Correct rate & $0.00 \%$ & $3.33 \%$ & $3.33 \%$ & $3.33 \%$ & $96.67 \%$ \\
\hline \multirow[t]{3}{*}{5} & Ave & $5.2010 \mathrm{E}+03$ & $4.6309 \mathrm{E}+03$ & $5.0515 E+03$ & $5.2326 \mathrm{E}+03$ & $5.3430 \mathrm{E}+03$ \\
\hline & CPU Time(sec) & 5.6052 & 4.1419 & 5.1839 & 6.8246 & 4.3532 \\
\hline & Correct rate & $63.33 \%$ & $20.00 \%$ & $10.00 \%$ & 83.33\% & $100.00 \%$ \\
\hline
\end{tabular}




\begin{tabular}{rlrrrrr}
\hline 6 & Ave & $2.2438 \mathrm{E}+03$ & $2.1788 \mathrm{E}+03$ & $2.4663 \mathrm{E}+03$ & $2.4687 \mathrm{E}+03$ & $\mathbf{2 . 7 7 9 4 E + 0 3}$ \\
& CPU Time(sec) & 4.3715 & 3.2339 & 3.9746 & 4.8534 & $\mathbf{3 . 1 1 2 6}$ \\
& Correct rate & $10.00 \%$ & $13.33 \%$ & $16.67 \%$ & $56.67 \%$ & $\mathbf{9 6 . 6 7 \%}$ \\
7 & Ave & $3.2231 \mathrm{E}+03$ & $3.6542 \mathrm{E}+03$ & $3.9975 \mathrm{E}+03$ & $4.3047 \mathrm{E}+03$ & $\mathbf{4 . 6 7 8 5 E + 0 3}$ \\
& CPU Time(sec) & 6.1227 & 4.9912 & 5.6239 & 6.1082 & $\mathbf{4 . 8 5 4 6}$ \\
& Correct rate & $0.00 \%$ & $16.67 \%$ & $23.33 \%$ & $73.33 \%$ & $\mathbf{9 3 . 3 3 \%}$ \\
& Ave & $7.5719 \mathrm{E}+03$ & $8.8193 \mathrm{E}+03$ & $1.0217 \mathrm{E}+04$ & $1.0726 \mathrm{E}+04$ & $\mathbf{1 . 1 0 0 5 E + 0 4}$ \\
& CPU Time(sec) & 10.5511 & 10.4806 & 9.7369 & 13.2446 & $\mathbf{8 . 1 4 6 9}$ \\
& Correct rate & $13.33 \%$ & $26.67 \%$ & $43.33 \%$ & $93.33 \%$ & $\mathbf{1 0 0 . 0 0 \%}$ \\
\hline
\end{tabular}

Table $2 p$ values calculated for the Wilcoxon rank-sum test via comparing LI-GWO vs LI-BBO, LI-BA,

LI-SMS, LI-ICA on eight test images ( $p \geq 0.05$ have been underlined)

\begin{tabular}{ccccc}
\hline LI-GWO vs & LI-BBO & LI-BA & LI-SMS & LI-ICA \\
\hline image 1 & $4.4677 \mathrm{E}-11$ & $2.0194 \mathrm{E}-07$ & $4.7382 \mathrm{E}-09$ & $3.1267 \mathrm{E}-02$ \\
image 2 & $1.2705 \mathrm{E}-05$ & $8.8611 \mathrm{E}-07$ & $4.4282 \mathrm{E}-12$ & $2.1095 \mathrm{E}-06$ \\
image 3 & $2.1982 \mathrm{E}-06$ & $1.6995 \mathrm{E}-08$ & $8.5600 \mathrm{E}-07$ & $6.3634 \mathrm{E}-05$ \\
image 4 & $1.5188 \mathrm{E}-12$ & $9.8401 \mathrm{E}-12$ & $1.1333 \mathrm{E}-11$ & $5.9503 \mathrm{E}-13$ \\
image 5 & $3.1335 \mathrm{E}-04$ & $1.9305 \mathrm{E}-09$ & $5.7426 \mathrm{E}-11$ & $2.1569 \mathrm{E}-02$ \\
image 6 & $1.0397 \mathrm{E}-09$ & $1.9381 \mathrm{E}-09$ & $1.3640 \mathrm{E}-08$ & $4.2099 \mathrm{E}-04$ \\
image 7 & $8.1708 \mathrm{E}-11$ & $5.1088 \mathrm{E}-08$ & $1.4658 \mathrm{E}-06$ & $3.8507 \mathrm{E}-02$ \\
image 8 & $1.9142 \mathrm{E}-10$ & $1.6851 \mathrm{E}-08$ & $2.1613 \mathrm{E}-06$ & $1.6080 \mathrm{E}-01$ \\
\hline
\end{tabular}

Table 1 shows the results for all images. As can be seen in this table, the LI-GWO outperforms the other algorithms on all the images in terms of the Ave and Correct rate. From Table 1, it turns out that the average CPU Time of LI-GWO provided better results than other algorithms across the majority of all images. For image 2 and image 5, however, the average CPU Time results of LI-BA are slightly better than other algorithms, and LI-GWO followed.

According to the $p$ values in Table 2, LI-GWO achieves significant improvement in images 1-7 compared to other algorithms. For image 8, the $p$ values show that the results of LI-GWO are significantly better in three out of four groups. Therefore, this is evidence that LI-GWO results are statistically significant and that it has not occurred by coincidence.

The results of the experiment with the original images shown in Figs. 3(a)-10(a) and the template images shown in Figs. 3(b)-10(b) are given. Original and template images processed by the lateral inhibition are shown in Figs. 3(c)-10(c) and Figs. 3(d)-10(d), respectively. It is obvious that the edges of these images are enhanced. So we can say that lateral inhibition has the ability to enhance the characters of image, which can improve the success rate of template matching. Figs. 3(e)-10(e) illustrate matching results for all images. From the matching results, it is clear that LI-GWO method can successfully find the precise location of the template fixed in the original image. Further experiments are given in Figs. 3(f)-10(f) to investigate the advantage of LI-GWO.

Figs. 3(f)-10(f) illustrate the averaged evolution curves of all algorithms dealing with all images over 30 independent runs. As can be seen from Figs. 3(f)-7(f) and Fig. 10(f), the LI-GWO has the fastest convergence rate. Through carefully looking at Figs. 8(f)-9(f), LI-ICA has a fast convergence initially towards the known maximum; however, it is outperformed by LI-GWO after 70 generations and 130 generations respectively. As the procedure proceeds, LI-GWO gets closer and closer to the maximum, 
while LI-ICA comes into being premature and traps into local maximum.

According to this comprehensive study, the superior exploration and local maxima avoidance of GWO are able to solve complicated template matching problem efficiently and accurately under different environments.

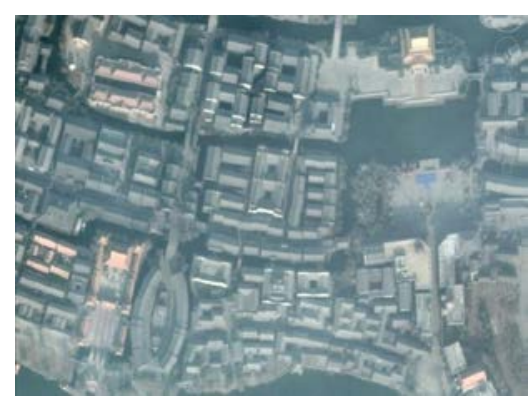

(a)

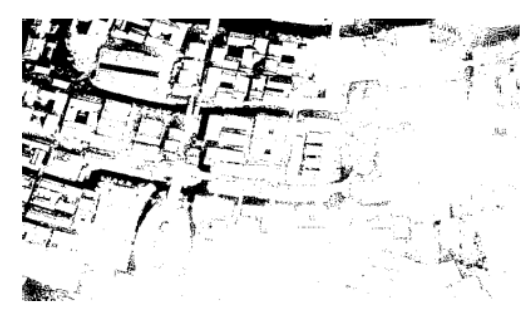

(c)

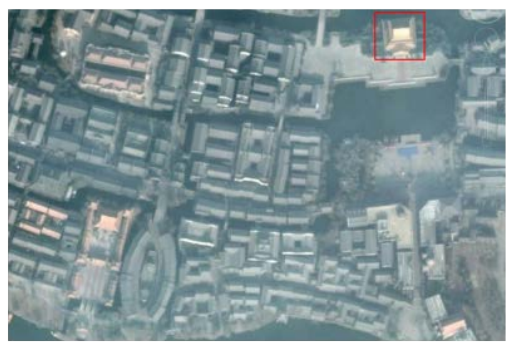

(e)

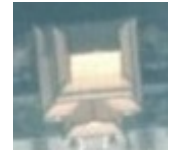

(b)

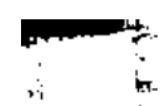

(d)

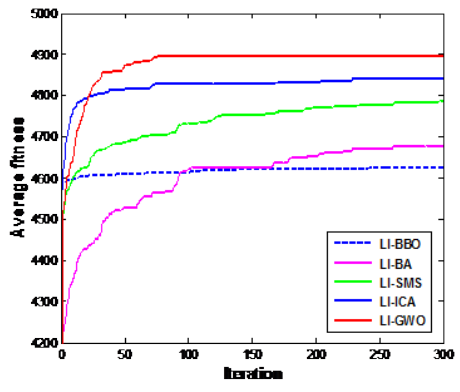

(f)

Fig. 3 Experimental results for image 1. (a) Original image $(749 \times 502)$. (b) Template image $(76 \times 73)$.

(c) Original image processed by the lateral inhibition. (d) Template image processed by the lateral inhibition.

(e) The final template matching result. (f) Evolution curves of experimental results for image 1.

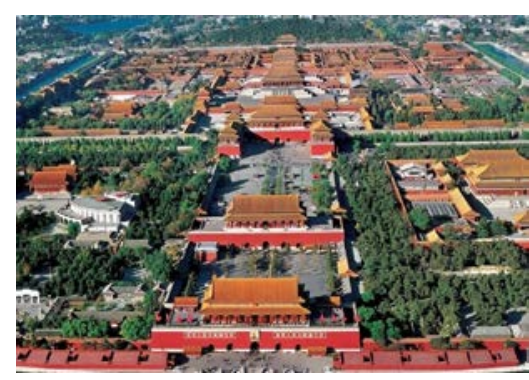

(a)

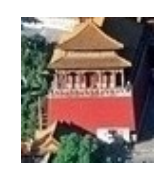

(b) 


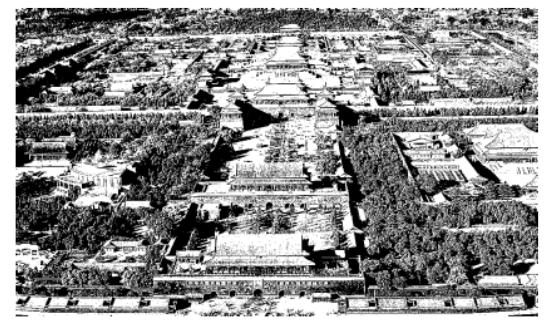

(c)

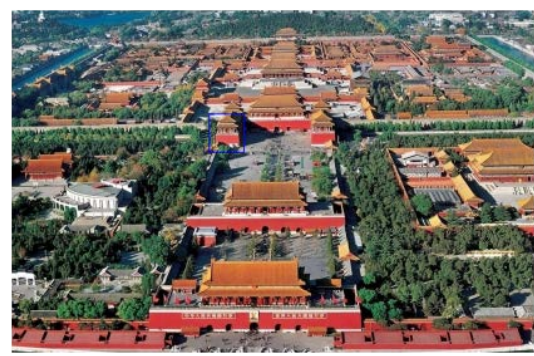

(e)

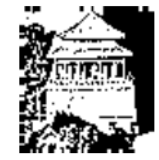

(d)

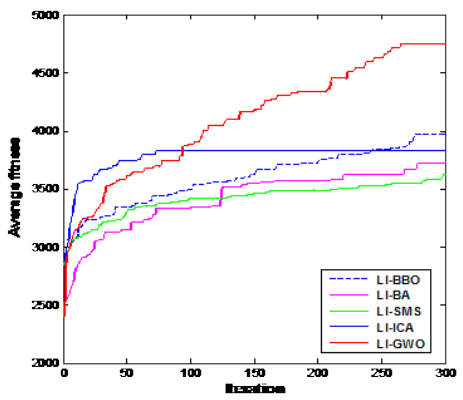

(f)

Fig. 4 Experimental results for image 2. (a) Original image $(1023 \times 672)$. (b) Template image (70× 77).

(c) Original image processed by the lateral inhibition. (d) Template image processed by the lateral inhibition.

(e) The final template matching result. (f) Evolution curves of experimental results for image 2.

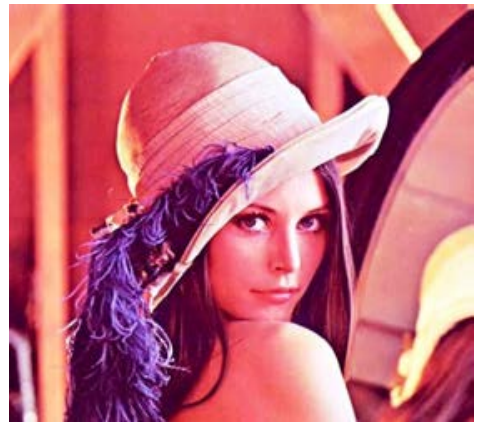

(a)

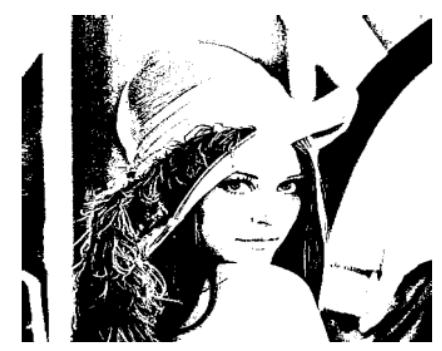

(c)

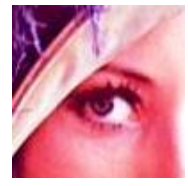

(b)

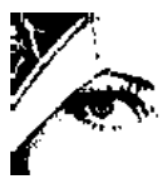

(d) 


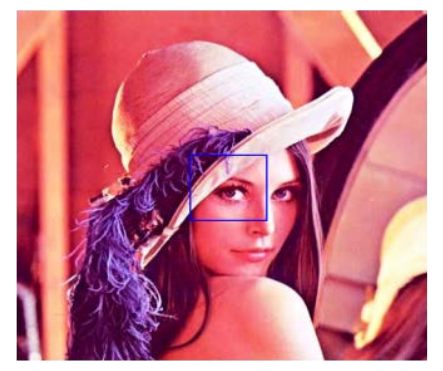

(e)

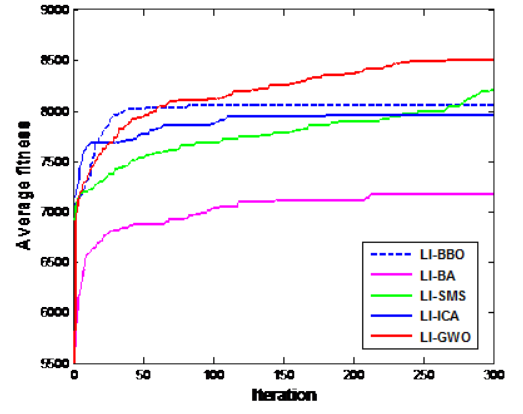

(f)

Fig. 5 Experimental results for image 3. (a) Original image (512× 512). (b) Template image $(97 \times 97)$.

(c) Original image processed by the lateral inhibition. (d) Template image processed by the lateral inhibition.

(e) The final template matching result. (f) Evolution curves of experimental results for image 3.

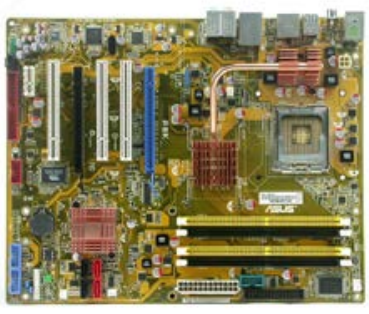

(a)

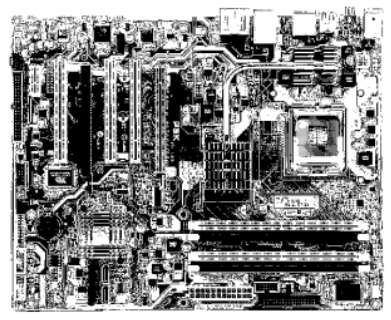

(c)

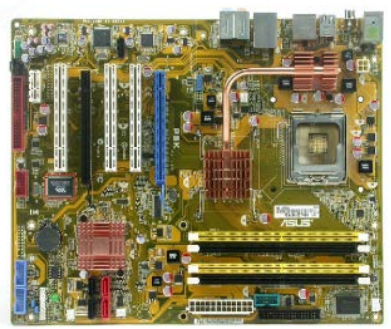

(e)

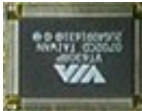

(b)

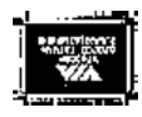

(d)

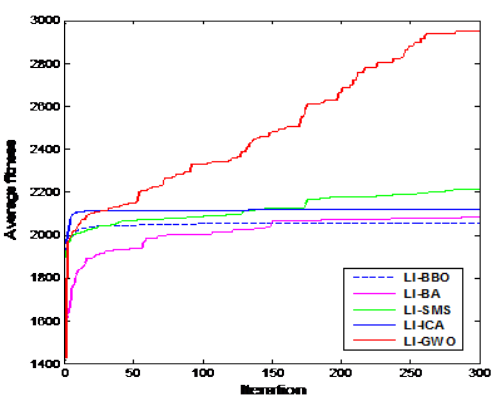

(f)

Fig. 6 Experimental results for image 4. (a) Original image $(1024 \times 768)$. (b) Template image $(68 \times 51)$.

(c) Original image processed by the lateral inhibition. (d) Template image processed by the lateral inhibition.

(e) The final template matching result. (f) Evolution curves of experimental results for image 4. 


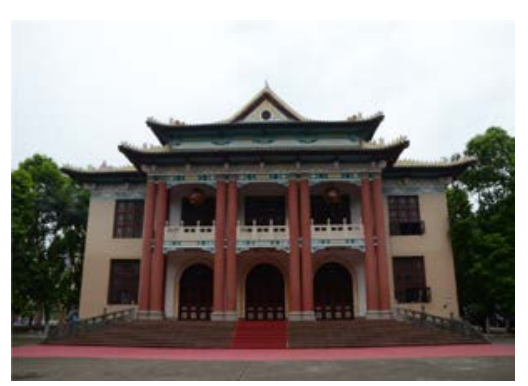

(a)

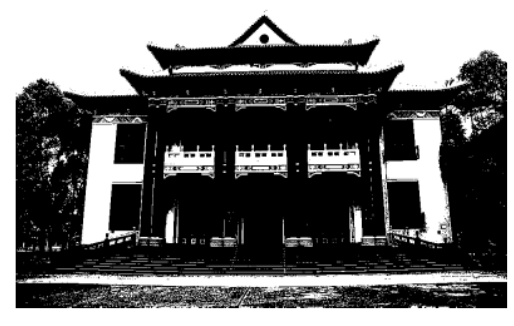

(c)

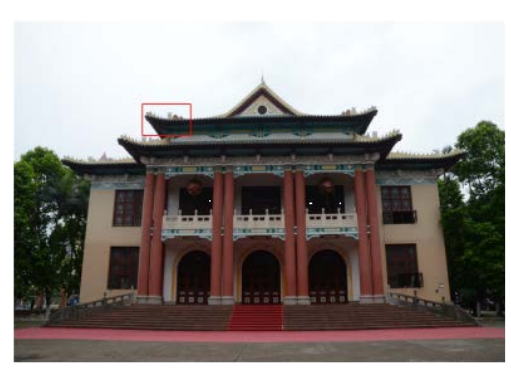

(e)

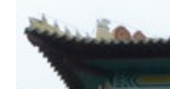

(b)

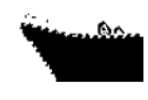

(d)

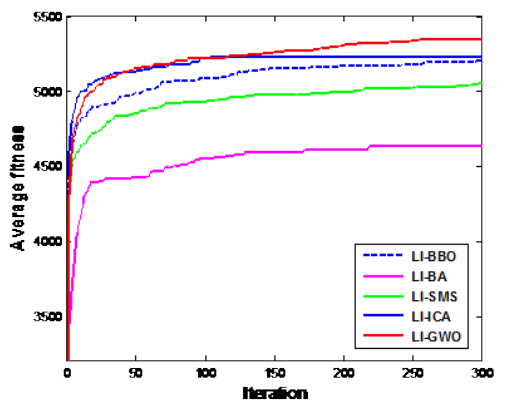

(f)

Fig. 7 Experimental results for image 5. (a) Original image $(980 \times 649)$. (b) Template image $(98 \times 61)$.

(c) Original image processed by the lateral inhibition. (d) Template image processed by the lateral inhibition.

(e) The final template matching result. (f) Evolution curves of experimental results for image 5.

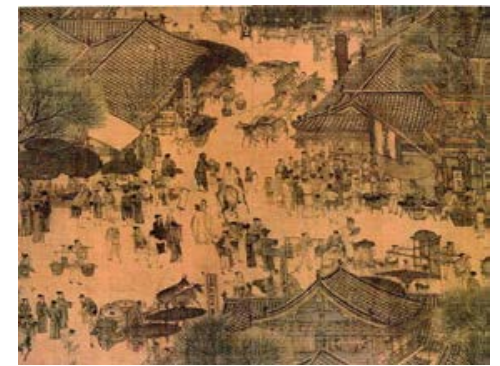

(a)

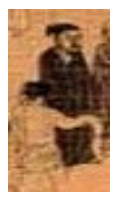

(b) 


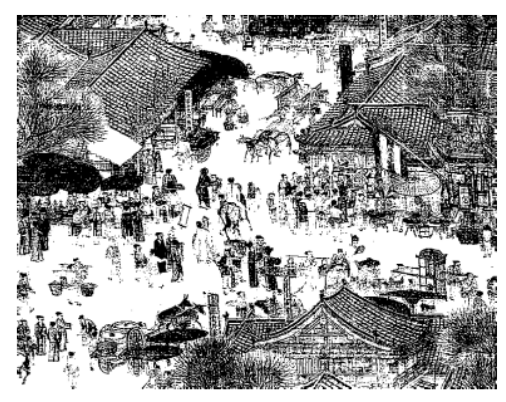

(c)

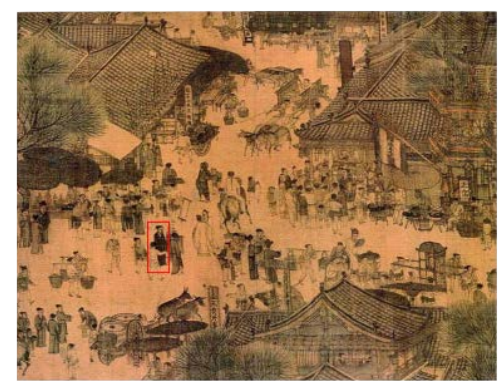

(e)

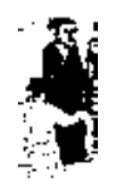

(d)

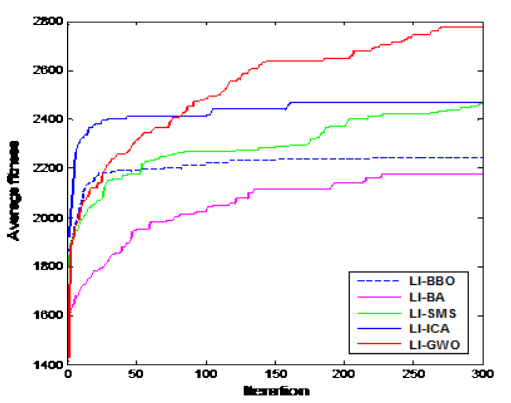

(f)

Fig. 8 Experimental results for image 6. (a) Original image $(832 \times 608)$. (b) Template image $(39 \times 85)$.

(c) Original image processed by the lateral inhibition. (d) Template image processed by the lateral inhibition.

(e) The final template matching result. (f) Evolution curves of experimental results for image 6 .

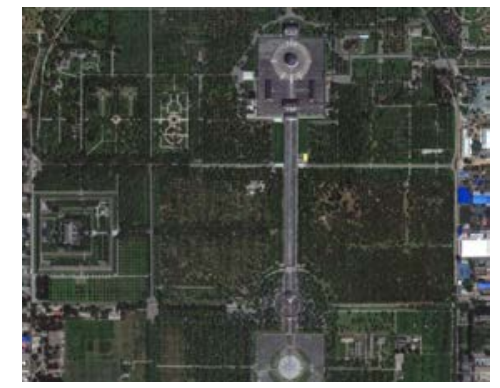

(a)

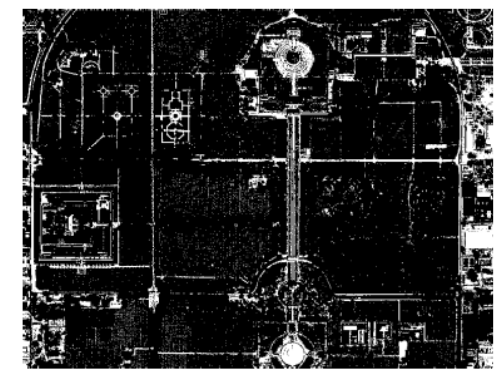

(c)

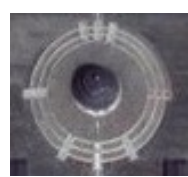

(b)

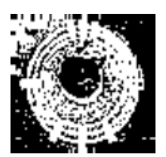

(d) 


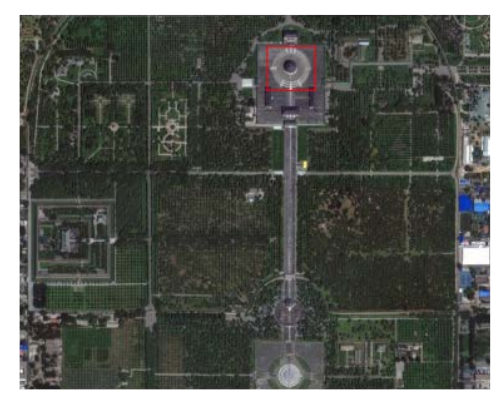

(e)

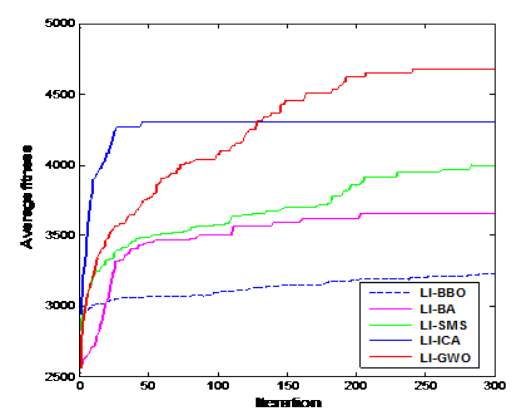

(f)

Fig. 9 Experimental results for image 7. (a) Original image ( $747 \times 595)$. (b) Template image $(78 \times 72)$.

(c) Original image processed by the lateral inhibition. (d) Template image processed by the lateral inhibition. (e) The final template matching result. (f) Evolution curves of experimental results for image 7.

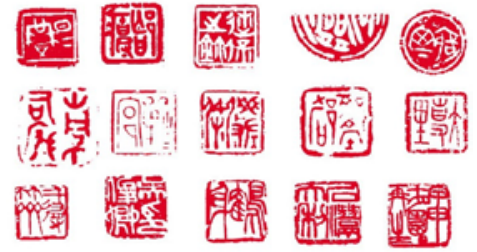

(a)

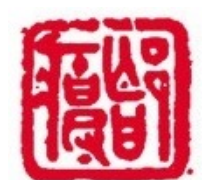

(b)

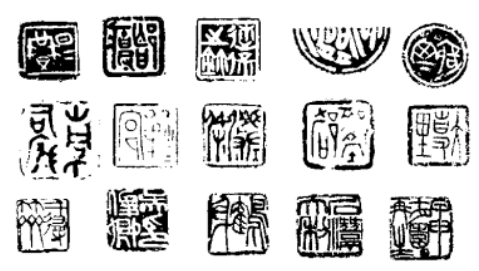

(c) (d)

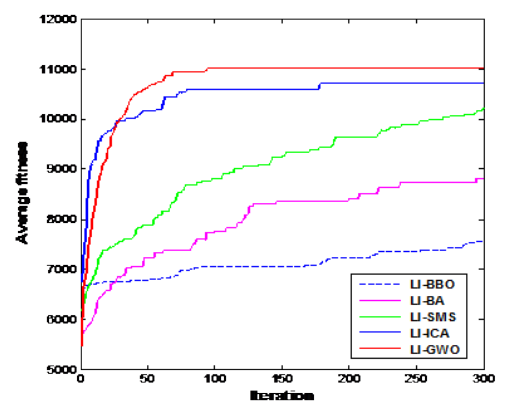

(f)

Fig. 10 Experimental results for image 8. (a) Original image $(730 \times 411)$. (b) Template image $(111 \times 107)$.

(c) Original image processed by the lateral inhibition. (d) Template image processed by the lateral inhibition.

(e) The final template matching result. (f) Evolution curves of experimental results for image 8. 


\subsection{Discussions}

Statistically speaking, the LI-GWO provides superior local optimal avoidance in all of the images (100\%), the less average CPU Time in six of all images (75\%) and the best accurate rate in all of the images (100\%). The reason why the GWO performed very well fitness value on template matching is due to the high local optima avoidance of this algorithm. According to equations of the GWO, half of the iterations are devoted to exploration of the search space (when $|A|>1$ ). This promotes exploration of the search space that leads to finding diverse template matching structures during optimization. Moreover, the $C$ parameter always randomly obliges the search agents to take random steps towards/outwards the prey. This mechanism is very helpful for resolving local optima stagnation even when the GWO is in the exploitation phase.

The results of this work show that although evolutionary algorithms have high exploration, the problem of template matching needs high local optima avoidance during the whole optimization process. This is because the search space is changed for every image in template matching. The results prove that the GWO is very effective in this regard. Moreover, Table 1 shows that LI-GWO usually consumes less time than LI-BBO, LI-BA, LI-SMS, and LI-ICA do. This is because LI-GWO neither adds complicated outside-world strategy nor changes the conventional algorithm framework.

The reason for the high accurate rate provided by the LI-GWO is that this algorithm is equipped with adaptive parameters to smoothly balance exploration and exploitation. Half of the iteration is devoted to exploration and the rest to exploitation. In addition, the GWO always saves the three best obtained solutions at any stage of optimization. Consequently, there are always guiding search agents for exploitation of the most promising regions of the search space. In other words, LI-GWO benefits from intrinsic exploitation guides, which also assist this algorithm to provide remarkable results.

\section{Conclusions and future works}

This paper presented a hybrid biological LI-GWO algorithm for template matching, which takes advantages of the efficiency of the GWO and the accuracy of lateral inhibition. GWO is a new evolutionary algorithm inspired by simulating hunting behavior and social leadership of grey wolves in nature. The application of lateral inhibition has improved the speed and stability of matching. Comparative experimental results between LI-GWO, LI-BBO, LI-BA, LI-SMS, and LI-ICA have proved that the proposed method performed better in solving template matching. It can be concluded that LI-GWO is a more effective and stable template matching method.

For the template matching, the following issues should be clarified in the future research. Firstly, there is some work which requires further investigation in order to reduce the computation time of template matching, and the convergence and the suitability of GWO for template matching should be further studied theoretically. On the other hand, new methods should be designed to solve template matching problem under complicated noisy environments.

\section{Acknowledgements}

This work is supported by National Science Foundation of China under Grants No. 61463007. 61563008 


\section{References}

[1] Brunelli R, Poggio T (1993) Face recognition: Features versus templates. IEEE Transactions on Pattern Analysis and Machine Intelligence 10: 1042-1052

[2] Lee Y, Hara T, Fujita H, Itoh S, Ishigaki T (2001) Automated detection of pulmonary nodules in helical CT images based on an improved template-matching technique. IEEE Transactions on Medical Imaging 20(7): 595-604

[3] Peng H, Long F, Chi Z (2003) Document image recognition based on template matching of component block projections. IEEE Transactions on Pattern Analysis and Machine Intelligence 25(9): 1188-1192

[4] Fu G, Zhao H, Li C, Shi L (2013) Road detection from optical remote sensing imagery using circular projection matching and tracking strategy. Journal of the Indian Society of Remote Sensing 41(4): 819-831

[5] Koutaki G, Yata K, Uchimura K, Kan M, Asai D, Takeba M (2013) Fast and high accuracy pattern matching using multi-stage refining eigen template. Frontiers of Computer Vision, IEEE, pp. 58-63

[6] Elboher E, Werman M (2013) Asymmetric correlation: a noise robust similarity measure for template matching. IEEE Transactions on Image Processing 22(8): 3062-3073

[7] Ma L, Sun Y, Feng N, Liu Z (2009). Image fast template matching algorithm based on projection and sequential similarity detecting. Intelligent Information Hiding and Multimedia Signal Processing, IEEE, pp. 957-960

[8] Duan H, Xu C, Liu S, Shao S (2010) Template matching using chaotic imperialist competitive algorithm. Pattern recognition letters 31(13): 1868-1875

[9] Zhang C, Duan H (2015) Biological lateral inhibition and Electimize approach to template matching. Optik-International Journal for Light and Electron Optics 126(7): 769-773

[10] Liu F, Duan H, Deng Y (2012) A chaotic quantum-behaved particle swarm optimization based on lateral inhibition for image matching. Optik-International Journal for Light and Electron Optics 123(21): 1955-1960

[11] Zhang Z, Duan H (2014) A hybrid Particle Chemical Reaction Optimization for biological image matching based on lateral inhibition. Optik-International Journal for Light and Electron Optics 125(19): 5757-5763

[12] Zhang JW, Wang GG (2012, November). Image matching using a bat algorithm with mutation. Applied Mechanics and Materials 203: 88-93

[13] Duan H, Deng Y, Wang X, Xu C (2013) Small and dim target detection via lateral inhibition filtering and artificial bee colony based selective visual attention. PloS one, 8(8): e72035

[14] Li B, Gong, L G, Li Y (2014) A novel artificial bee colony algorithm based on internal-feedback strategy for image template matching. The Scientific World Journal, Volume 2014 (2014), Article ID 906861, 14 pages

[15] Li B (2016) An Evolutionary Approach for Image Retrieval Based on Lateral Inhibition. Optik-International Journal for Light and Electron Optics, doi:10.1016/j.ijleo.2016.02.056

[16] Wang X, Duan H, Luo D (2013) Cauchy biogeography-based optimization based on lateral inhibition for image matching. Optik-International Journal for Light and Electron Optics 124(22): 5447-5453

[17] Cuevas E, Echavarría A, Zaldívar D, Pérez-Cisneros M (2013) A novel evolutionary algorithm 
inspired by the states of matter for template matching. Expert Systems with Applications 40(16): 6359-6373

[18] Huang L, Duan H, Wang Y (2014) Hybrid bio-inspired lateral inhibition and imperialist competitive algorithm for complicated image matching. Optik-International Journal for Light and Electron Optics 125(1): 414-418

[19] Mirjalili S, Mirjalili SM, Lewis A (2014) Grey wolf optimizer. Advances in Engineering Software 69: 46-61

[20] Mirjalili S (2015) How effective is the Grey Wolf optimizer in training multi-layer perceptrons. Applied Intelligence 43(1): 150-161

[21] Zhang S, Zhou Y (2015) Grey Wolf Optimizer Based on Powell Local Optimization Method for Clustering Analysis. Discrete Dynamics in Nature and Society, Volume 2015 (2015), Article ID 481360, 17 pages

[22] Song X, Tang L, Zhao S, Zhang X, Li L, Huang J, Cai W (2015) Grey Wolf Optimizer for parameter estimation in surface waves. Soil Dynamics and Earthquake Engineering 75: 147-157

[23] Sulaiman MH, Mustaffa Z, Mohamed MR, Aliman O (2015) Using the gray wolf optimizer for solving optimal reactive power dispatch problem. Applied Soft Computing 32: 286-292

[24] Zhu A, Xu C, Li Z, Wu J, Liu Z (2015) Hybridizing grey wolf optimization with differential evolution for global optimization and test scheduling for 3D stacked SoC. Journal of Systems Engineering and Electronics 26(2): 317-328

[25] Korayem L, Khorsid M, Kassem SS (2015) Using Grey Wolf Algorithm to Solve the Capacitated Vehicle Routing Problem. In IOP Conference Series: Materials Science and Engineering, 83(1): 012014

[26] El-Fergany AA, Hasanien HM (2015) Single and Multi-objective Optimal Power Flow Using Grey Wolf Optimizer and Differential Evolution Algorithms. Electric Power Components and Systems, 43(13): 1548-1559

[27] Gupta E, Saxena A (2016) Grey wolf optimizer based regulator design for automatic generation control of interconnected power system. Cogent Engineering, doi:10.1080/23311916.2016.1151612

[28] Pan TS, Dao TK, Chu SC (2015) A Communication Strategy for Paralleling Grey Wolf Optimizer. In Genetic and Evolutionary Computing, Springer International Publishing, pp. 253-262

[29] Hartline HK (1938) The response of single optic nerve fibers of the vertebrate eye to illumination of the retina. American Journal of Physiology--Legacy Content 121(2): 400-415

[30] Fang Z, Dawei Z, Ke Z (2007) Image pre-processing algorithm based on lateral inhibition. Electronic Measurement and Instruments, IEEE, pp. 701-705

[31] Yang XS (2014) Nature-inspired optimization algorithms. Elsevier press.

[32] Derrac J, García S, Molina D, Herrera F (2011) A practical tutorial on the use of nonparametric statistical tests as a methodology for comparing evolutionary and swarm intelligence algorithms. Swarm and Evolutionary Computation 1(1): 3-18

[33] Gibbons JD, Chakraborti S (2011) Nonparametric statistical inference. Springer Berlin Heidelberg, pp 977-979

[34] Hollander M, Wolfe DA, Chicken E (2013) Nonparametric statistical methods. John Wiley and Sons. 